\title{
Hepatitis B virus X protein modulates remodelling of minichromosomes related to hepatitis $B$ virus replication in HepG2 cells
}

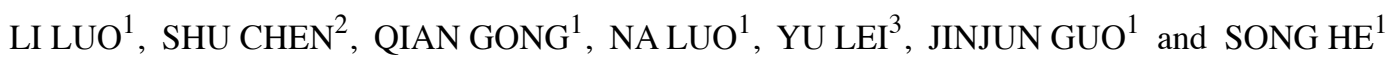 \\ Departments of ${ }^{1}$ Gastroenterology and ${ }^{2}$ Haematology, The Second Affiliated Hospital of Chongqing Medical University, \\ Chongqing; ${ }^{3}$ Institute for Viral Hepatitis, Chongqing Medical University, Chongqing, P.R. China
}

Received June 7, 2012; Accepted August 14, 2012

DOI: $10.3892 /$ ijmm.2012.1165

\begin{abstract}
Hepatitis B virus (HBV) covalently closed circular DNA (cccDNA) is organised into minichromosomes by histone and non-histone proteins. Remodelling of minichromosomes is crucial for the regulation of HBV replication, which is dependent on the presence of the hepatitis $B$ virus $X$ protein $(\mathrm{HBx})$. However, the mechanisms of $\mathrm{HBx}$-dependent $\mathrm{HBV}$ replication remain obscure. The objective of this study was to investigate the mechanism of $\mathrm{HBx}$-dependent $\mathrm{HBV}$ replication through the pathway of chromatin remodelling. The role of HBx was investigated by transfecting human HepG2 cells with the linear full-length HBV genome (wild-type) or HBx-deficient mutant HBV DNA (HBx mutant). Our results showed that although the formation of cccDNA was not affected by $\mathrm{HBx}$, HBV replication, transcription and antigen secretion were all significantly reduced, resulting from the absence of $\mathrm{HBx}$. The acetylation, mono-methylation and phosphorylation of
\end{abstract}

Correspondence to: Dr Song He or Dr Jinjun Guo, Department of Gastroenterology, The Second Affiliated Hospital of Chongqing Medical University, 74 Linjiang Road, Yuzhong, Chongqing 400010, P.R. China

E-mail: hedoctor65@sina.com

E-mail: guojinjun1972@163.com

Abbreviations: cccDNA, covalently closed circular DNA; ChIP, chromatin immunoprecipitation; CREB, cAMP-response element protein; DNMTs, DNA methyltransferases; DL, doublestranded linear; ELISA, enzyme-linked immunosorbent assay; $\mathrm{GFP}$, green fluorescence protein; HBV, hepatitis B virus; HBx, hepatitis $\mathrm{B}$ virus $\mathrm{X}$ protein; HCC, hepatocellular carcinoma; HBsAg, hepatitis B surface antigen; $\mathrm{HBeAg}$, hepatitis B e-antigen; HATs, histone acetyltransferases; HDACs, histone deacetylases; HMTs, histone methyltransferases; IP, immunoprecipitation; IgG, immunoglobulin G; MAPK, mitogen-activated protein kinase; OD, optical density; pgRNA, pregenomic RNA; PVDF, polyvinylidene fluoride; PBS, phosphate-buffered saline; rcDNA, relaxed circular DNA; RIPA, radioimmunoprecipitation assay; SS, single-strand; SD, standard deviation

Key words: hepatitis $\mathrm{B}$ virus, hepatitis $\mathrm{B}$ virus $\mathrm{X}$ protein, $\mathrm{HBx}$ mutant, histone $\mathrm{H} 3$, histone modifications
cccDNA-bound histone $\mathrm{H} 3$ were associated with HBV replication. In addition, the levels of cccDNA-bound methylated, phosphorylated and acetylated histone $\mathrm{H} 3$ decreased sharply in HBx mutant HBV DNA. HBx modulated not only the status of acetylation but also the methylation and phosphorylation of histone $\mathrm{H} 3$ bound to the cccDNA during HBV replication in HepG2 cells. These findings suggest that HBx plays an important role in modulating the remodelling of minichromosomes related to HBV replication and it may regulate viral replication through the pathway of chromatin remodelling.

\section{Introduction}

Hepatitis B virus (HBV) infection is a major risk factor for the development of severe liver diseases including hepatocellular carcinoma (HCC) (1). Hepatitis B virus X protein ( $\mathrm{HBx})$ is a $17-\mathrm{kDa}$ protein encoded by the $3.2-\mathrm{kb}$ HBV genome (2). Previous studies have indicated that $\mathrm{HBx}$ is oncogenic and can regulate HBV replication and transcription (3). One study was performed using a plasmid carrying a greater-than-unit-length HBV genome (payw1.2) (4) and a HBx-deficient plasmid containing a stop codon at amino acid position 7 (payw1.2.7) (5) transfected into HepG2 cells, respectively. Results have shown that the absence of HBx can induce a $65 \%$ reduction in HBV replication (6), and trans-complementation of $\mathrm{HBx}$ for the HBx-deficient plasmid can restore replication to wild-type levels (6-8). Another advanced study performed with the hydrodynamic injection of an $\mathrm{HBx}$-deficient plasmid into mice showed the same results in vivo $(6,9,10)$. These results demonstrated that $\mathrm{HBx}$ has an important role in modulating HBV replication.

However, to date, the mechanisms of HBx-dependent HBV replication are not very clear. HBV covalently closed circular DNA (cccDNA), the main replicative intermediate of $\mathrm{HBV}$, is the template for transcription of all viral RNAs including pregenomic RNA (pgRNA) (11). Nuclear cccDNA, predominately from relaxed circular DNA (rcDNA) (12), is organised by histone and non-histone proteins into a viral minichromosome chromatin-like structure $(11,13,14)$. Changes in the nucleosome (the basic unit of chromatin) structure and DNA-histone contacts may result in the remodelling of minichromosomes (14). Chromatin remodelling is closely associated with histone modifications, especially with modifications of histone $\mathrm{H} 3$ and 
H4. These histone modifications may influence the structure of nucleosomes directly, and provide DNA binding sites for other proteins $(13,15)$. Previous studies have shown that $\mathrm{HBx}$ can regulate $\mathrm{HBV}$ replication and transcription. Multiple signal transduction pathways and proteins may be involved in HBX-dependent HBV replication and transcription $(16,17)$. These signalling mediators may have as their terminal target chromatin remodelling (18). Thus, it is hypothesized that the mechanisms of HBx-dependent HBV replication may involve the chromatin remodelling pathway.

Our previous study demonstrated that acetylation, methylation and phosphorylation of cccDNA-bound histone $\mathrm{H} 3$ occurs in HepG2 cells that are replicating wild-type HBV genome and that these histone modifications are associated with HBV replication (19). Our present study was designed to investigate the mechanism of $\mathrm{HBX}$-dependent $\mathrm{HBV}$ replication through the pathway of chromatin remodelling. We established two in vitro replication models by transfecting human hepatoma HepG2 cells with the linear full-length HBV genome (wild-type) or the HBx-deficient mutant HBV DNA (HBx mutant) and investigated the regulation of $\mathrm{HBx}$ on replication, transcription and antigen secretion, and in particular, on the methylation, phosphorylation and acetylation of histone $\mathrm{H} 3$ bound to the cccDNA in chromatin during HBV replication in HepG2 cells.

\section{Materials and methods}

Plasmid. The plasmid pUC-HBV1.0, which contains full-length wild-type HBV genome and HindIII/SapI and SacI/SapI restriction sites, was constructed as previously described (19). The HBx mutant plasmid pUC-HBV1.0.X7 that contains a stop codon (CAA-UAA) at amino acid 7 of $\mathrm{HBx}$ was derived from plasmid pUC-HBV1.0 by site-directed mutagenesis $(5,8)$. Briefly, mutagenic primers were designed using primer design software developed by Stratagene. The forward primer was 5'-CTAGGCTGTGCTGCTAACTGGATCCTGCG-3' (mutated nucleotides underlined) and the reverse primer was 5'-CGCA GGATCCAGTTAGCAGCACAGCCTAG-3'. Using plasmid pUC-HBV1.0 as a template, the mutant products were amplified by the high-fidelity enzyme Premix PrimeSTAR ${ }^{\circledR}$ HS (Takara) through polymerase chain reaction (PCR). The PCR products were then digested with DpnI enzyme (Fermentas), transformed into competent DH-5 $\alpha$ cells, which were plated on LB plates containing ampicillin $(100 \mu \mathrm{g} / \mathrm{ml})$. Four white colonies selected randomly were prepared, and the plasmid DNA was extracted and digested with HindIII and SacI enzymes (Takara). The plasmid, whose digested products were determined to be correct, was then sequenced (Takara) to confirm the mutation. All plasmids were prepared and purified using the Endotoxin-Free Plasmid Maxi kit (Tiangen Biotech, Co., Ltd.).

Cell culture and DNA transfection. Human hepatoma HepG2 cells were cultured in 6-well plates (Gibco) with high glucose DMEM containing 10\% fetal calf serum (Hyclone) under 5\% $\mathrm{CO}_{2}$ at $37^{\circ} \mathrm{C}$. The linear full-length $\mathrm{HBV}$ genome and the $\mathrm{HBx}$ mutant HBV DNA were released from plasmids pUC-HBV1.0 and pUC-HBV1.0.X7, respectively, by SapI enzyme (MBI) digestion and gel-purified by a DNA gel extraction kit (Promega). HBV DNA was transiently transfected into HepG2 cells using PolyJet ${ }^{\mathrm{TM}}$ reagent (SignaGen Laboratories). Briefly,
HepG2 cells were seeded at a density of $1.0 \times 10^{6}$ cells in 6-well plates. Twenty-four hours later, cells at 70-80\% confluence were transfected with HBV DNA $(1.5 \mu \mathrm{g})$ and PolyJet ${ }^{\mathrm{TM}}$ reagent $(4 \mu \mathrm{l})$. HepG 2 cells were transfected with the linear full-length HBV genome (wild-type) or the HBx-deficient mutant HBV DNA (HBx mutant). A green fluorescent protein (GFP) expression vector $(0.5 \mu \mathrm{g})$ was included in each transfection to assess transfection efficiency. After transfection, the cell culture medium was changed daily. The mean transfection efficiency was approximately $40 \%$. A negative control with no plasmid transfected into the HepG2 cells was set up in each independent experiment.

Immunoprecipitation (IP) and western blot analysis detection of HBx. Cells were harvested at 48 and $96 \mathrm{~h}$ post-transfection and lysed in radioimmunoprecipitation assay (RIPA) buffer (50 mM Tris, $150 \mathrm{mM} \mathrm{NaCl}, 1 \% \mathrm{NP}-40,0.5 \%$ sodium deoxycholate and $0.1 \%$ SDS). Following centrifugation to remove cellular debris, the supernatants were incubated with Protein $G$ Plus-Agarose (Santa Cruz Biotechnology, Inc.) and rabbit antiHBx polyclonal antibody (Abcam) for immunoprecipitation. The precipitated complexes were subjected to sodium dodecyl sulphate-polyacrylamide gel electrophoresis and transferred to polyvinylidene fluoride (PVDF) membranes. After blocking with $5 \%$ bovine serum albumin (Sigma) for $1 \mathrm{~h}$, the membrane was incubated overnight at $4^{\circ} \mathrm{C}$ with rabbit anti-HBx polyclonal antibody $(1: 1,000)$, followed by incubation with an anti-rabbit secondary antibody conjugated to horseradish peroxidase (1:2,000; Pierce). The bound antibodies were visualised using an ECL chemiluminescence system $(6,20)$.

Analysis of secreted HBV antigens. Culture supernatants collected from transfected cells at different time points were clarified by centrifugation at 3,000 rpm for $15 \mathrm{~min}$ and stored at $-20^{\circ} \mathrm{C}$ until used. Hepatitis B surface antigen (HBsAg) and hepatitis B e-antigen (HBeAg) were detected by an enzymelinked immunosorbent assay (ELISA) kit (Shanghai, KeHua) according to the manufacturer's instructions. The absorbance of the contents in each well was determined at the wavelength of $450 \mathrm{~nm}$. Positive and negative control sera were included in each assay. The results were expressed as mean optical density (OD) values [mean \pm standard deviation (SD)].

Southern blot analyses. At 48 and 96 h post-transfection, capsid-associated HBV DNA was extracted as described previously (8). Transfected cells were washed with cold phosphate-buffered saline (PBS) and lysed in 1\% NP lysis buffer [50 mM Tris (pH 7.4), 1 mM EDTA, 1\% NP-40, and $100 \mathrm{mM}$ $\mathrm{NaCl}$ ]. After centrifugation for $1 \mathrm{~min}$ at $12,000 \mathrm{rpm}$ at $4^{\circ} \mathrm{C}$, the supernatants were treated with $100 \mu \mathrm{g} / \mathrm{ml}$ DNase I (Promega) for $30 \mathrm{~min}$ at $37^{\circ} \mathrm{C}$ and then $0.5 \mathrm{mg} / \mathrm{ml}$ proteinase $\mathrm{K}$ at $50^{\circ} \mathrm{C}$ for $2 \mathrm{~h}$. Viral DNA released from lysed cores were extracted with phenol/chloroform, precipitated with ethanol, and dissolved in Tris-EDTA. Nuclear HBV cccDNA was extracted as described $(15,21)$. Simply, transfected cells were lysed in cell lysis buffer [50 mM Tris- $\mathrm{HCl}$ (pH 8.0), 1 mM EDTA, 0.2\% NP-40, and $150 \mathrm{mM} \mathrm{NaCl}$ ]. After centrifugation for $10 \mathrm{~min}$ at 12,000 rpm at $4^{\circ} \mathrm{C}$, the precipitate was resuspended in nuclear lysis buffer $(6 \% \mathrm{SDS}, 100 \mathrm{mM} \mathrm{NaOH})$ and incubated for $30 \mathrm{~min}$ at $37^{\circ} \mathrm{C}$. The lysates were then neutralised with potassium acetate 
(pH 4.8) and centrifuged for $10 \mathrm{~min}$ at $12,000 \mathrm{rpm}$ at $4^{\circ} \mathrm{C}$. Nucleic acids were purified by phenol/chloroform extraction and ethanol precipitation. The replicative intermediates of capsid-associated HBV DNA and nuclear HBV cccDNA were detected by Southern blotting. Ten micrograms of capsidassociated HBV DNA and HBV cccDNA was separated on a $1 \%$ agarose gel, transferred onto a positively charged nylon membrane, and hybridised with a ${ }^{32} \mathrm{P}$-labelled full-length HBV DNA probe (Takara). The membrane was then washed and exposed to film at $-80^{\circ} \mathrm{C}(22)$.

Quantitative analysis of capsid-associated HBV DNA. At 24, 48, 72 and $96 \mathrm{~h}$ after transfection, capsid-associated HBV DNA in HepG2 cells was extracted and quantitated by real-time PCR using TaqMan probes. The primers were 5'-AGAAACAACA CATAGCGCCTCAT-3' (forward) and 5'-TGCCCCATGCTG TAGATCTTG-3' (reverse). The TaqMan probe was 5'-FAMTGTGGGTCACCATATTCTTGGG-TAMER-3' (6). The cycling parameters, performed with an Applied Biosystems 7300 sequence detection system, were as followed: $95^{\circ} \mathrm{C}$ for $30 \mathrm{sec}$, then $40 \mathrm{cycles}$ of $95^{\circ} \mathrm{C}$ for $5 \mathrm{sec}$ and $60^{\circ} \mathrm{C}$ for $31 \mathrm{sec}$. The plasmid pUC-HBV1.0 was diluted over a range of $10^{9}-10^{3}$ copies and used as a standard. The results were expressed as the number of DNA copies/cell (mean $\pm \mathrm{SD})$.

Quantitative analysis of $H B V$ cccDNA. HBV cccDNA was quantified by real-time PCR as previously described (15). HBV cccDNA extracted from transfected HepG2 cells was treated with Plasmid-Safe DNase (Epicentre) at $37^{\circ} \mathrm{C}$ for $1 \mathrm{~h}$ to remove the open circular duplex HBV DNA and single-strand HBV DNA. The primers were 5'-CTCCCCGTCTGTGCCTTCT-3' (forward) and 5'-GCCCCAAAGCCACCCAAG-3' (reverse). The probes were 5'-GTTCACGGTGGTCTCCATGCAA CGT-FAM-3' and 5'-ROX-AGGTGAAGCGAAGTGCACAC GGACC-PO4-3' $(15,19)$. Real-time PCR experiments were performed with a LightCycler (Roche) as followed: pre-denaturation for $10 \mathrm{~min}$ at $95^{\circ} \mathrm{C}$, then 45 cycles of $10 \mathrm{sec}$ at $95^{\circ} \mathrm{C}$, $5 \mathrm{sec}$ at $58^{\circ} \mathrm{C}, 10 \mathrm{sec}$ at $63^{\circ} \mathrm{C}$, and $20 \mathrm{sec}$ at $72^{\circ} \mathrm{C}$. Standard curves were prepared as described in the quantitative analysis of capsid-associated HBV DNA by real-time PCR. The results were expressed as the number of DNA copies/cell (mean \pm SD).

Quantitative analysis of HBV pregenomic RNA (pgRNA). For pgRNA analysis, total cellular RNA was extracted with TRIzol reagent (Invitrogen) from transfected HepG2 cells at different time points post-transfection. RNA concentration and purity were determined by ultraviolet spectrometry. The RNA samples were treated with RNase-Free DNase (Promega) at $37^{\circ} \mathrm{C}$ for $30 \mathrm{~min}$, and reverse transcribed into cDNA using PrimeScript ${ }^{\circledR}$ RT reagent kit (Takara). Each cDNA was quantified by real-time PCR using SYBR ${ }^{\circledR}$ Premix Ex Taq ${ }^{\mathrm{TM}}$ II kit (Takara). The specific primers for pgRNA were 5'-GCCTTAGAGTCTCCTGAGCA-3' (forward) and 5'-GAGGGAGTTCTTCTTCTAGG-3' (reverse) (21), and for GAPDH were 5'-GAAGGTGAAGGTCGGAGTC-3' (forward) and 5'-GAAGATGGTGATGGGATTTC-3' (reverse). Amplification of GAPDH cDNA was used to normalise the RNA samples.

cccDNA chromatin immunoprecipitation (ChIP) assays. ChIP assays were performed with an EZ-Magna ChIP assay kit (no. 17-408; Millipore) according to the manufacturer's specifications. Briefly, at 24, 48, 72 and 96 h post-transfection, HepG2 cells were cross-linked by incubation with $1 \%$ formaldehyde for $10 \mathrm{~min}$ at room temperature, which was terminated with $10 \mathrm{X}$ glycine by incubation at room temperature for $5 \mathrm{~min}$. The collected cells were washed twice with cold PBS and lysed with cell lysis buffer by incubation on ice for $15 \mathrm{~min}$. The cell pellet was then resuspended in nuclear lysis buffer and sonicated to generate 300-400 bp DNA fragments. After centrifugation, $50 \mu \mathrm{l}$ of each of the supernatants (1x10 ${ }^{6}$ cell equivalents) was diluted 1:10 with ChIP dilution buffer and a $1 \%$ volume of the mixture was taken as input. The chromatin was then subjected to immunoprecipitation for $14-16 \mathrm{~h}$ at $4^{\circ} \mathrm{C}$ with anti-H3 (no. 06-755), anti-acetylated histone $\mathrm{H} 3$ at lysines 9 and 14 (no. 06-599), anti-monomethylated histone $\mathrm{H} 3$ at lysine 4 (no. 07-436), anti-phosphorylated histone $\mathrm{H} 3$ at serine 10 (no. 04-817; all were from Millipore) antibodies, and $20 \mu \mathrm{l}$ of fully suspended protein A magnetic beads. Immunoprecipitation with the relevant nonspecific immunoglobulin $\mathrm{G}$ ( $\mathrm{IgG}$ ) was included in each experiment as a negative control. After reversal of the cross-linking, DNA from the antibody-bound and input fractions was isolated and treated with plasmid-safe DNase at $37^{\circ} \mathrm{C}$ for $1 \mathrm{~h}$. Purified ChIP cccDNA and input DNA were then analysed by PCR and real-time PCR using cccDNA-selective primers and probes (15). HBV cccDNA-selective primers were HBV P23 (5'-CTGAATCCCGCGGACGACCC-3') (1443-1462), and P24 (5'-ACCCAAGGCACAGCTTGGAGG-3') (1891-1871), which were specific to the $\mathrm{HBV}$ precore-core promoter region to distinguish cccDNA from rcDNA $(25,26)$. The PCR reaction was performed with the high-fidelity enzyme Premix PrimeSTAR ${ }^{\circledR} \mathrm{HS}$ as follows: 35 cycles of $10 \mathrm{sec}$ at $98^{\circ} \mathrm{C}, 5 \mathrm{sec}$ at $60^{\circ} \mathrm{C}$ and $1 \mathrm{~min}$ at $72^{\circ} \mathrm{C}$. The PCR products were analysed by electrophoresis. ChIP cccDNA and input DNA were absolutely quantified by real-time PCR as described above. Results were expressed as the percentage of input DNA.

Statistical analysis. The data presented from at least 3 separate experiments were expressed as the means \pm SD. Statistical comparisons of the continuous variables between the 2 groups were performed using the nonparametric Wilcoxon rank-sum test (SPSS 19.0 software). P-values of $<0.05$ were assigned to indicate statistically significant results.

\section{Results}

$H B x$ is required for enhancement of $H B V$ replication in HepG 2 cells. To detect the effect of $\mathrm{HBx}$ on HBV replication, equivalent number of HepG2 cells were transfected with the linear full-length HBV genome (wild-type) or the HBx mutant. Capsid-associated HBV DNA was extracted from HepG2 cells at $24,48,72$ and $96 \mathrm{~h}$ post-transfection and quantified by realtime PCR. The levels of capsid-associated HBV DNA from the wild-type HBV-transfected cells were $39.1 \pm 2.9$ copies/cell at $48 \mathrm{~h}$ which declined to $10.5 \pm 1.4$ copies/cell at $96 \mathrm{~h}$, while levels from the HBx mutant-transfected cells were $36.6 \pm 2.5$ copies/ cell at $48 \mathrm{~h}$ which decreased to $4.9 \pm 1.1$ copies/cell at $96 \mathrm{~h}$ (Fig. 1A). Between 24 and 48 h, when HBV replication reached peak levels, the levels of capsid-associated DNA in the HBx mutant-transfected cells were slightly affected by the lack of 

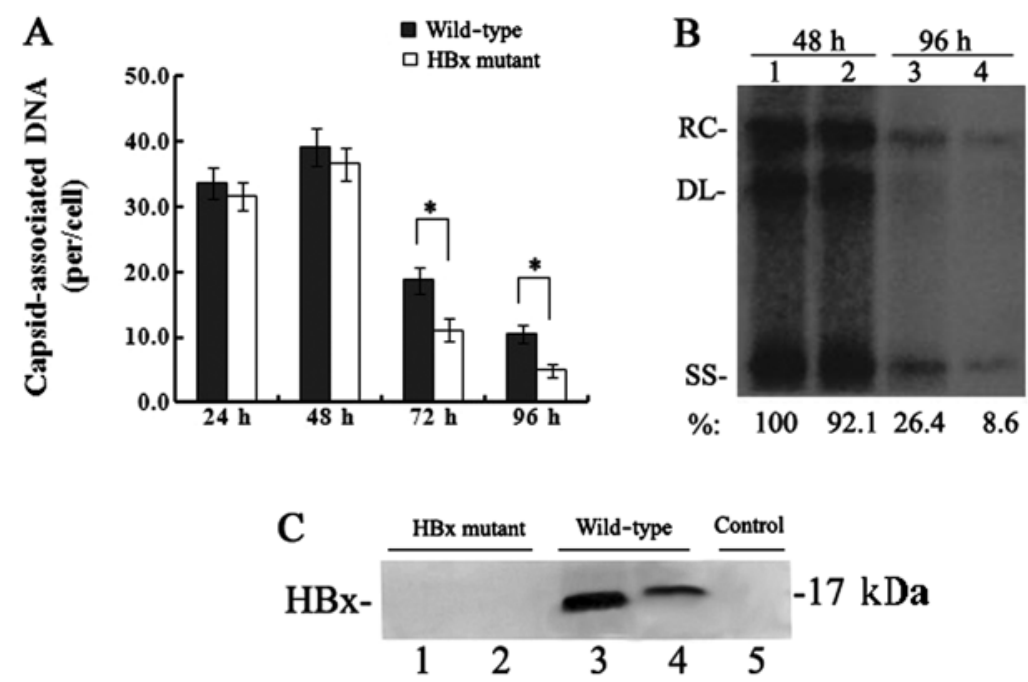

Figure 1. Effect of HBx on HBV replication in transfected HepG2 cells. (A) Quantification of capsid-associated HBV DNA by real-time PCR at 24, 48, 72 and $96 \mathrm{~h}$ post-transfection. Results are expressed as the number of capsid-associated DNA copies per cell (mean \pm SD) from 3 independent experiments. Statistical significance is designated with asterisks above the brackets. (B) Southern blot analysis of capsid-associated viral DNA replicative intermediates at 48 and $96 \mathrm{~h}$ after transfection. Signal intensity of the single-stranded (SS) band underneath the double-stranded linear (DL) HBV DNA band was quantified with Quantity One Analysis software (Bio-Rad). The band corresponding to the DL HBV DNA was not included in the quantitative analysis, as this DNA might be partially derived from transfected input DNA. Lanes 1 and 3, the wild-type HBV genome; lanes 2 and 4, HBx mutant HBV DNA. The number at the bottom of each lane represents the relative levels of HBV DNA replicative intermediates, with those levels detected in the wild-type HBV-transfected cells at $48 \mathrm{~h}$ set to $100 \%$, and levels measured in the HBx mutant-transfected cells are compared to those in the wild-type HBV-transfected cells from three independent experiments; RC, relaxed circular; DL, double-stranded linear; and SS, single-stranded forms. (C) Representative IP/western blotting. A negative control with no plasmid transfected into HepG2 cells (lane 1); HepG2 cells transfected with the linear HBx-deficient mutant HBV DNA (HBx mutant) and the full-length HBV genome (wild-type) were harvested at 48 and 96 h, and analysed by IP/western blotting for HBx protein. Lanes 1 and 3, 48 h; lanes 2 and 4, 96 h.

HBx $(\mathrm{P}>0.05)$; however, the levels of capsid-associated HBV DNA were significantly reduced at 72 and in particular at $96 \mathrm{~h}$, which showed a $50-70 \%$ reduction $(\mathrm{P}<0.05)$ as compared to those levels in the wild-type HBV-transfected cells. The results of the Southern blotting by which replicative intermediates of capsid-associated DNA were detected with a ${ }^{32} \mathrm{P}$-labelled full-length HBV DNA probe were consistent with those of the real-time PCR quantitation of capsid-associated DNA (Fig. 1B). A sensitive IP/western blot assay was used to detect the expression of HBx in transfected HepG2 cells. HBx was below the limit of detection by IP/western blot assay in the HBx mutant-transfected cells, but was detected at both 48 and $96 \mathrm{~h}$ in the wild-type HBV-transfected cells, with the expression level of HBx at $96 \mathrm{~h}$ much lower than that at $48 \mathrm{~h}$ (Fig. 1C). Together, these results demonstrate that $\mathrm{HBx}$ is required for the enhancement of HBV replication in HepG2 cells.

$H B x$ affects the secretion of the $H B V$ antigen. To investigate the effect of HBx on antigen secretion, the secretion of HBsAg and $\mathrm{HBeAg}$ from cell culture supernatants was tested by ELISA at 4 time-points. The results showed that the secretion of HBV antigens was detected in cells transfected with both wild-type and HBx mutant HBV DNA. At $24 \mathrm{~h}$ after transfection, there was no significant difference in the secretion of HBsAg and HBeAg between the 2 types of HBV DNA (P>0.05) (Fig. 2). With the extension of time after transfection, differences gradually appeared. In particular, at $96 \mathrm{~h}$ the secretion of HBsAg and HBeAg from HBx mutant-transfected cells was 55.7\% ( $\mathrm{P}<0.05)$ (Fig. 2A) and 55.2\% ( $\mathrm{P}<0.05)$ (Fig. 2B), respectively, of that from wild-type $\mathrm{HBV}$-transfected cells. These results indicate that the lack of $\mathrm{HBx}$ reduces the secretion of viral antigens in HepG2 cells.
HBx does not affect $H B V$ cccDNA formation, but upregulates the transcription of $p g R N A$. The HBV cccDNA extracted from HepG2 cells at 24, 48, 72 and 96 h post-transfection was purified after treatment with plasmid-safe DNase and quantitatively analysed by real-time PCR. The level of cccDNA in wild-type HBV-transfected cells was $38.4 \pm 3.1$ copies/cell at $48 \mathrm{~h}$ which decreased to $6.9 \pm 1.5$ copies/cell at $96 \mathrm{~h}$, and the level in the HBx mutant-transfected cells was $36.5 \pm 2.7$ copies/ cell at $48 \mathrm{~h}$ which declined to $5.8 \pm 1.2$ copies/cell at $96 \mathrm{~h}$ (Fig. 3A). When HBV replication reached a peak between 24 and $48 \mathrm{~h}$ or when levels of both nuclear cccDNA and HBV replication were reduced severely at 72 and $96 \mathrm{~h}$, the mean copies of cccDNA per HepG2 cell were similar with the 2 types of HBV genome. The results of the real-time PCR quantitation of HBV cccDNA were confirmed by Southern blotting by which nuclear cccDNA was detected (Fig. 3B). Altogether, these results demonstrate that $\mathrm{HBx}$ is not required for HBV cccDNA formation.

Since $\mathrm{HBx}$ is not required for the formation of $\mathrm{HBV}$ cccDNA, the effect of HBx on a downstream step, pgRNA transcription, was investigated. At 4 time-points posttransfection, the extracted RNA was reverse transcribed and quantified by real-time PCR. These results showed that levels of pgRNA were slightly lower between 24 and $48 \mathrm{~h}$ after transfection in the HBx mutant DNA ( $\mathrm{P}>0.05)$. However, differences gradually appeared after this time. In particular, at $96 \mathrm{~h}$, the levels of pgRNA were reduced by $50-70 \%$ in the absence of HBx, compared to levels in the cells transfected with wild-type HBV DNA $(\mathrm{P}<0.05)$ (Fig. 3C). Taken together, these findings indicate that $\mathrm{HBx}$ upregulates pgRNA transcription without affecting the formation of HBV cccDNA. 

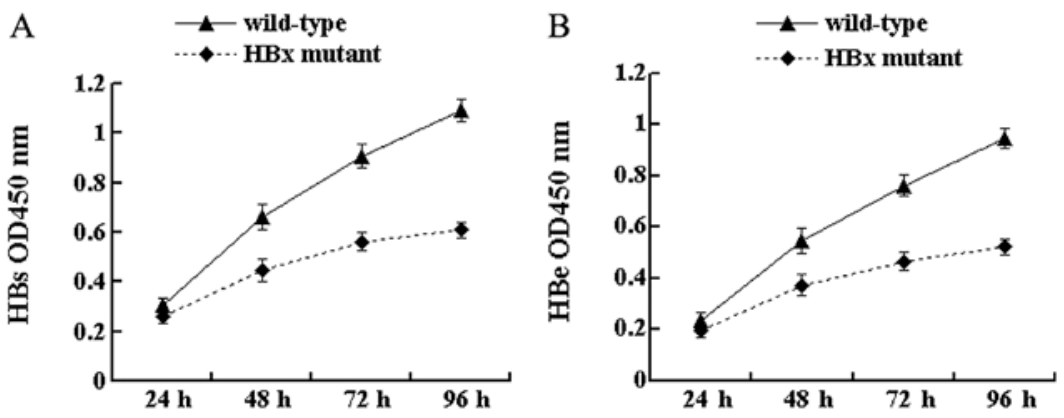

Figure 2. Effect of HBx on HBV antigen secretion in HepG2 cells transfected with the linear full-length HBV genome (wild-type) or the HBx-deficient mutant HBV DNA (HBx mutant). Culture supernatants were collected at 24, 48, 72 and $96 \mathrm{~h}$ after transfection and detected by ELISA. (A) HBsAg; (B) HBeAg. Results are expressed as the OD values (mean $\pm \mathrm{SD}$ ) measured at a wavelength of $450 \mathrm{~nm}$ from 3 independent experiments. SD, standard deviation.

A

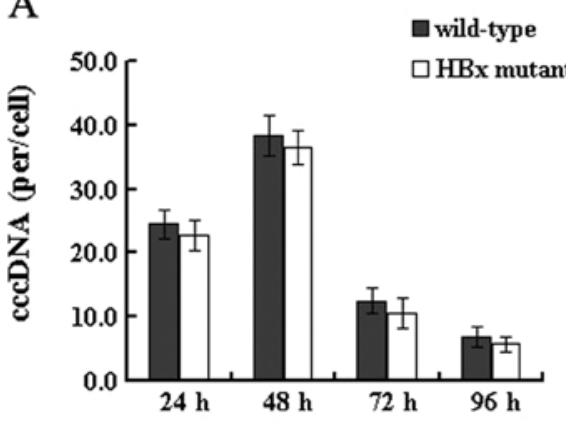

B
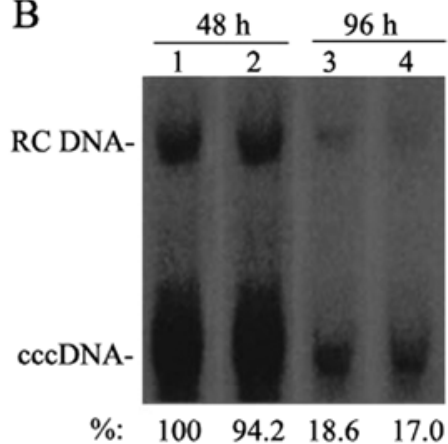

C

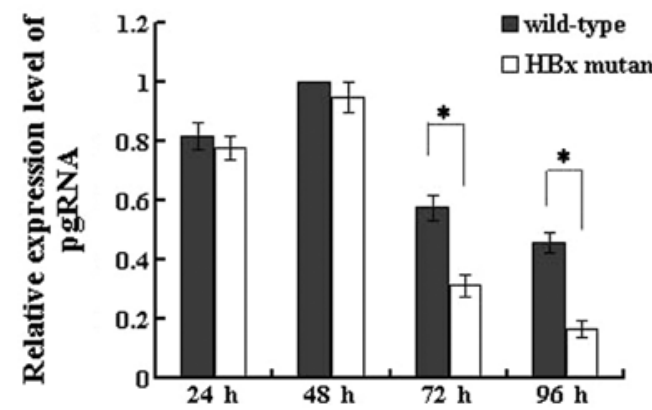

Figure 3. Effect of HBx on cccDNA formation and transcription of pgRNA in HepG2 cells transfected with the linear full-length HBV genome (wild-type) or the HBx-deficient mutant HBV DNA (HBx mutant). (A) Quantification of HBV cccDNA by real-time PCR at 4 time-points post-transfection. Results are expressed as the number of cccDNA copies/cell (mean \pm SD) from 3 independent experiments (B) Southern blot analysis of nuclear cccDNA at 48 and $96 \mathrm{~h}$ after transfection. Signal intensity of the cccDNA band was quantified with Quantity One Analysis software. Lanes 1 and 3, the wild-type HBV genome; lanes 2 and 4, HBx mutant HBV DNA. The number at the bottom of each lane represents the relative levels of cccDNA, with those detected in the wild-type HBVtransfected cells at $48 \mathrm{~h}$ set to $100 \%$, and levels measured in the HBx mutant HBV-transfected cells are compared to those in the wild-type HBV-transfected cells from 3 independent experiments. (C) Specific primers were used to quantitate relative HBV pregenomic RNA (pgRNA), and GAPDH amplification was used to normalise for each RNA sample. The level of pgRNA detected in the wild-type HBV-transfected cells at $48 \mathrm{~h}$ post-transfection was set to 1.0 , and the level measured in HBx mutant HBV-transfected cells was compared to that in the wild-type HBV-transfected cells. Results are expressed as the mean \pm SD from 3 independent experiments. Statistical significance is designated with asterisks above the bracket. pgRNA, pregenomic RNA.

$H B x$ regulates remodelling of the minichromosome related to $H B V$ replication. Since $\mathrm{HBx}$ has proven to be crucial for HBV replication and transcription, we sought to study whether HBx affects remodelling of the minichromosome related to HBV replication. Immunoprecipitated cccDNA from HepG2 cells at 24, 48, 72 and $96 \mathrm{~h}$ after transfection was amplified by PCR and quantified by real-time PCR. In cells transfected with the wild-type HBV genome (Fig. 4), the cccDNA-bound histone H3 was highly acetylated, hypermethylated and hyperphosphorylated simultaneously between 24 and $48 \mathrm{~h}$ post-transfection when the level of HBV replication reached a peak. Levels of cccDNA-bound acetylated, mono-methylated and phosphorylated histone $\mathrm{H} 3$ peaked at $4.53 \pm 0.71,3.31 \pm 0.62$ and $3.62 \pm 0.59$, in units of $\%$ input DNA, respectively. At $72 \mathrm{~h}$, when the HBV replication declined, the levels of cccDNA-bound acetylated and phosphorylated histone $\mathrm{H} 3$ were sharply reduced compared to those at $48 \mathrm{~h}($ all $\mathrm{P}<0.05)$. Both the acetylation and phosphorylation of cccDNA-bound $\mathrm{H} 3$ histone decreased by $72 \mathrm{~h}$ to $2.24 \pm 0.31$ and $1.71 \pm 0.22$ in units of \% input DNA, respectively (all $\mathrm{P}<0.05$ ). However, levels of cccDNA-bound methylated $\mathrm{H} 3$ did not change appreciably at $72 \mathrm{~h}$, and the values in units of \% input DNA were 
A

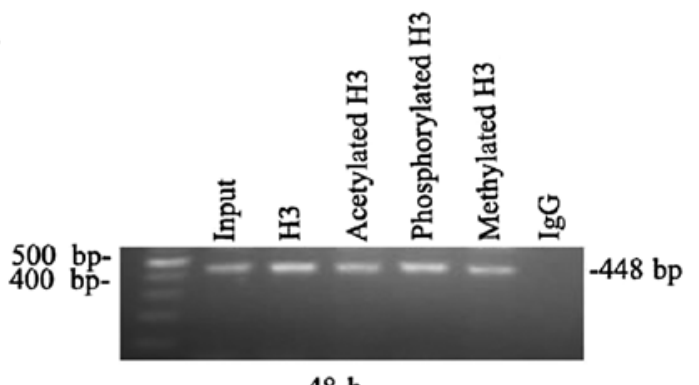

B

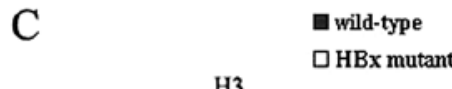

$48 \mathrm{~h}$
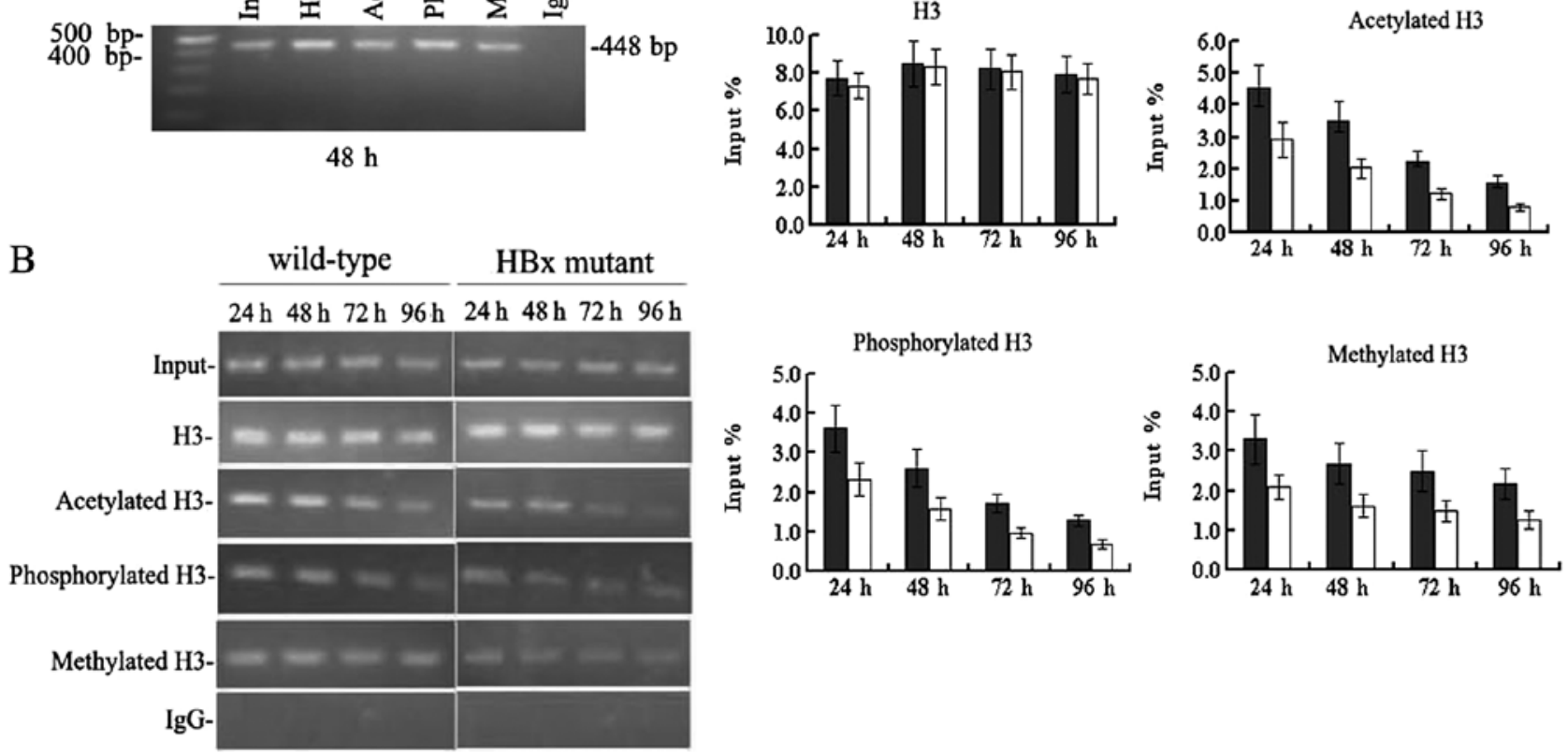

Figure 4. Regulation of HBx on the remodelling of the minichromosome related to HBV replication. (A) HepG2 cells were transfected with the linear fulllength HBV genome (wild-type). Chromatin prepared at $48 \mathrm{~h}$ post-transfection was immunoprecipitated with the relevant control IgG or the specific anti-H3, anti-acetylated histone $\mathrm{H} 3$ at lysines 9 and 14, anti-monomethylated histone $\mathrm{H} 3$ at lysine 4 and anti-phosphorylated histone $\mathrm{H} 3$ at serine 10 antibodies, and analysed by semi-quantitative PCR. (B and C) HepG2 cells were transfected with the linear full-length HBV genome (wild-type) or the HBx-deficient mutant HBV DNA (HBx mutant). Chromatin prepared at 24, 48, 72 and $96 \mathrm{~h}$ post-transfection was immunoprecipitated with the relevant control IgG or specific anti$\mathrm{H} 3$, anti-acetylated histone $\mathrm{H} 3$ at lysines 9 and 14, anti-monomethylated histone $\mathrm{H} 3$ at lysine 4 and anti-phosphorylated histone $\mathrm{H} 3$ at serine 10 antibodies, and analysed by (B) semi-quantitative PCR and (C) real-time quantitative PCR with HBV cccDNA-selective primers. The results are expressed as percentages of input chromatin (mean $\pm \mathrm{SD}$ ) from 3 independent experiments.

$2.69 \pm 0.52$ and $2.49 \pm 0.51$ at 48 and $72 \mathrm{~h}$, respectively $(\mathrm{P}>0.05)$ (Fig. 4B and C). These results indicate that the acetylation and phosphorylation of cccDNA-bound histones $\mathrm{H} 3$ are dynamic, but the methylation of cccDNA-bound histone $\mathrm{H} 3$ is relatively stable at lysine 4. Acetylation, methylation and phosphorylation of cccDNA-bound histone $\mathrm{H} 3$ paralleled $\mathrm{HBV}$ replication in HepG2 cells.

Next we investigated the epigenetic changes in cells transfected with the HBx mutant HBV genome. cccDNA-bound histone $\mathrm{H} 3$ was not only rapidly hypo-acetylated but also hypophosphorylated and hypomethylated (Fig. 4B and C). At $48 \mathrm{~h}$ post-transfection, cccDNA-bound acetylated, phosphorylated and methylated histone $\mathrm{H} 3$ were reduced by $40-50 \%$ in the absence of HBx compared to those levels in cells transfected with the wild-type HBV DNA (all $\mathrm{P}<0.05$ ). The acetylation, methylation, and phosphorylation of cccDNA-bound $\mathrm{H} 3$ were $2.01 \pm 0.32,1.61 \pm 0.28$ and $1.58 \pm 0.29$ in units of $\%$ input DNA, respectively. At $96 \mathrm{~h}$, levels of the above-mentioned modifications of histone $\mathrm{H} 3$ were very low. These results were in agreement with the reduction observed in $\mathrm{HBV}$ replication (Fig. 1A and B), pgRNA transcription (Fig. 3C) and antigen secretion (Fig. 2). Together, these findings demonstrate that HBx can affect the methylation, phosphorylation and acetylation of cccDNA-bound histone $\mathrm{H} 3$ during $\mathrm{HBV}$ replication in HepG2 cells. In other words, HBx can regulate the remodelling of the minichromosomes related to $\mathrm{HBV}$ replication in HepG2 cells. Considering that epigenetic modifications of cccDNAbound $\mathrm{H} 3$ histone parallel HBV replication, it is now possible to state that $\mathrm{HBx}$ may regulate viral replication through the pathway of chromatin remodelling.

\section{Discussion}

In our study, the HBx-deficient plasmid pUC-HBV1.0.X7 was successfully constructed by site-directed mutagenesis, and two in vitro replication models by transfecting HepG2 cells with the linear full-length HBV genome (wild-type) or the HBx-deficient mutant HBV DNA (HBx mutant) were established successfully. We found that although the formation of HBV cccDNA was not affected by HBx, there was a dramatic reduction in HBV replication, pgRNA transcription and antigen secretion in the absence of HBx compared to levels in cells transfected with the wild-type HBV genome. In addition, the levels of cccDNA-bound methylated, phosphorylated and acetylated histone $\mathrm{H} 3$ decreased sharply in $\mathrm{HBx}$ mutant HBV DNA. These results suggest that HBx is required for the enhancement of HBV replication and transcription. HBx modulates not only the status of acetylation but also the methylation and phosphorylation of cccDNA-bound histone $\mathrm{H} 3$ related to HBV replication in HepG2 cells. 
Although it is not completely clear why HBV cccDNA formation is not affected by HBx, our study has demonstrated that $\mathrm{HBx}$ plays an importance role in regulating methylation and phosphorylation in addition to acetylation of cccDNAbound histone $\mathrm{H} 3$ during HBV replication and elucidated the mechanism of HBx-dependent HBV replication through the pathway of chromatin remodelling. It has been shown that HBx does not directly bind to DNA sequences but is recruited onto the chromatin through its ability to interact with various cellular partners and proteins (23). The recruitment of $\mathrm{HBx}$ onto the cccDNA parallels the dynamic changes of cccDNAbound acetylated H3 (21). HBx favours the aceylation of histone $\mathrm{H} 3$ bound to cccDNA and modulates HBV replication and transcription $(15,24)$. However, our present study has shown that the methylation, phosphorylation and acetylation of cccDNA-bound histone $\mathrm{H} 3$ paralleled HBV replication in HepG2 cells. The cccDNA-bound histone H3 was highly acetylated, hypermethylated and hyperphosphorylated when the level of HBV replication reached a peak; while the levels of cccDNA-bound acetylated and phosphorylated histone $\mathrm{H} 3$ were also reduced when the HBV replication declined, although the levels of cccDNA-bound methylated $\mathrm{H} 3$ did not change appreciably. But why acetylation and phosphorylation of cccDNA-bound histone $\mathrm{H} 3$ decreased over time apart from methylation remains obscure. The difference in HBV replication between the groups of wild-type and HBx-mutant appeared only at $72 \mathrm{~h}$ post-transfection whereas the difference in epigenetic modifications was already detected $24 \mathrm{~h}$ posttransfection. The possible interpretations are that the absence of HBx may change chromatin structure and DNA-histone contacts, resulting in remodelling of minichromosomes; the decline of HBV replication may be associated with the early decrease in histone modifications. HBx modulates not only the status of acetylation but also the methylation and phosphorylation of histone $\mathrm{H} 3$ bound to the cccDNA during HBV replication. Therefore, one of the mechanisms of HBX-dependent HBV replication may be that HBX influences epigenetic modifications, leading to chromatin remodelling.

Histone lysine methylation is closely related to gene transcription, which has a different effect at different aminoterminal residues. Methylation of lysines 9 and 27 in histone $\mathrm{H} 3$ correlates with gene repression, whereas methylation of lysines 4, 36 and 79 in histone $\mathrm{H} 3$ correlates with activation $(27,28)$. Our study suggested that HBx modulates the status of hisone $\mathrm{H} 3$ methylation. The possible explanation from some studies is that SET and MYND domain-containing protein 3 (SMYD3) is one of the methyltransferases for $\mathrm{H} 3$ lysine 4 $(29,30)$. RNA polymerase II is recruited to the promoter region of SMYD3 gene by HBx, leading to the enhancement of the expression of SMYD3 and cellular activities of HMTs at H3 lysine $4(30,31)$. Histone modifications, as well as modifications of the DNA, can influence chromatin structure, induce the remodelling of chromatin and consequently result in gene silencing (27). HBx can increase the activities of total DNA methyltransferases (DNMTs) by upregulation of DNMT1, DNMT3A1 and DNMT3A2, and can selectively promote regional hypermethylation of specific tumour-suppressor genes (32). In the cytoplasm, HBx increases DNMT1 by activating the Ras signalling pathway $(32,33)$ and/or by inhibiting p53 function $(32,34)$. HBx interacts with DNMT3A to trigger epigenetic modifications at different loci, thus regulating the transcription of target genes. For example, HBx recruits DNMT3A to the promoter region of ML1F and IL4R, inducing inhibition of ML1F and IL4R and regional hypermethylation. In contrast, HBx separates DNMT3A from the promoter of IGFBP-3 and CDH6, resulting in activation of IGFBP-3 and CDH6 and downregulation of DNA methylation (35).

Histone $\mathrm{H} 3$ serine 10 phosphorylation is important for transcriptional activation and chromosome condensation occurring during mitosis, meiosis, apoptosis and DNA damage (27). Our study has shown that HBx modulates the status of histone $\mathrm{H} 3$ phosphorylation. HBx can also promote nuclear protein serine phosphorylation and increases pgRNA encapsidation and HBV DNA synthesis, which may be attributable to the activation of the $\mathrm{HBx}$-induced signal transduction pathways including the core protein serine kinases (36). A rapid phosphorylation of histone $\mathrm{H} 3$ at serine 10 is induced by the early response genes c-fos and c-myc when the Ras mitogen-activated protein kinase (MAPK) signalling pathway is stimulated $(37,38)$. Modifications of histones are quite complex and can interplay with each other due to the cross-talk between them (39). The activation of Aurora-kinase-B-mediated phosphorylation of H3 serine 10 may serve as a 'phos-methyl switch', leading to the separation of HP1 from heterochromatin while maintaining H3K9 methylation during mitosis $(39,40)$. Acetylation of histones at lysines 9 and 14 can serve as a prelude to transcriptional activation, whereas methylation of histones at lysine 9 can lead to gene silencing and formation of heterochromatin. These modifications may influence the ability of serine 10 to be phosphorylated and vice versa (41).

Histone acetyltransferases (HATs) and histone deacetylases (HDACs) are responsible for the steady-state balance of acetylation modification of histones, although this balance can be affected by HBx protein. Cellular histone acetytransferases p300, CBP, PCAF/GCN5, and the histone deactylases HDAC1 and hSirtl are recruited with different kinetics onto cccDNA in HBV replication $(13,21)$. A previous study demonstrated that there is no $\mathrm{HBx}$ recruited onto cccDNA in cells replicating the HBx mutant HBV virus (21). cccDNA-bound histones are rapidly hypoacetylated in the absence of $\mathrm{HBx}$, and the recruitment of p300 is severely impaired while the recruitment of the histone deacetylases HDACl and hSirtl is increased and occurs earlier (21). Our study also demonstrated that HBx modulates the status of histone $\mathrm{H} 3$ acetylation. The possible explanation is that HBx affects the expression of important cellular genes resulting from its transcriptional transactivation and transrepression properties. $\mathrm{HBx}$ can directly interact with the acetyltransferase $\mathrm{p} 300 / \mathrm{CBP}$ complex in coordination to enhance the activity of CREB to promote transcription, leading to activation of the acetylated histone state of the target cellular genes $(18,42)$. HBx recruits HDAC1 to the promoter of IGFBP-3 and induces the formation of the Sp1/HDAC1 complex, resulting in deacetylation of $\mathrm{Sp} 1$ and inhibition of the transcription of IGFBP-3 (43).

In summary, HBx plays a pivotal role in HBV replication and transcription. Specifically, $\mathrm{HBx}$ affects not only the status of acetylation but also methylation and phosphorylation of cccDNA-bound histone $\mathrm{H} 3$ during HBV replication in HepG2 cells. HBx may modulate HBV replication through the pathway of minichromosome remodelling related to $\mathrm{HBV}$ 
replication. Further study is required to ascertain whether HBx modulates HBV replication by affecting the recruitment of other histone-modifying enzymes bound to the cccDNA in addition to HATs and HDACs. In this regard, our research provides experimental evidence for elucidating the mechanism of HBx-dependent HBV replication through the pathway of chromatin remodelling and identified the HBx protein as a new target for antiviral treatment at the level of cccDNA.

\section{Acknowledgements}

This study was supported by grants from the National Natural Science Foundation of China (grant no. 30872249) and key program of Medical Science of Chongqing Health Bureau (grant no. 2010-1-37).

\section{References}

1. Dienstag JL: Hepatitis B virus infection. N Engl J Med 359: 1486-1500, 2008.

2. Seeger $C$ and Mason WS: Hepatitis B virus biology. Microbiol Mol Biol Rev 64: 51-68, 2000.

3. Murakami S: Hepatitis B virus X protein: a multifunctional viral regulator. J Gastroenterol 36: 651-660, 2001.

4. Scaglioni PP, Melegari M and Wands JR: Posttranscriptional regulation of hepatitis $B$ virus replication by the precore protein. J Virol 71: 345-353, 1997.

5. Melegari M, Scaglioni PP and Wands JR: Cloning and characterization of a novel hepatitis B virus $\mathrm{x}$ binding protein that inhibits viral replication. J Virol 72: 1737-1743, 1998.

6. Keasler VV, Hodgson AJ, Madden CR and Slagle BL: Enhancement of hepatitis $B$ virus replication by the regulatory $X$ protein in vitro and in vivo. J Virol 81: 2656-2662, 2007.

7. Bouchard MJ, Wang LH and Schneider RJ: Calcium signaling by HBx protein in hepatitis B virus DNA replication. Science 294: 2376-2378, 2001.

8. Tang H, Delgermaa L, Huang F, et al: The transcriptional transactivation function of $\mathrm{HBx}$ protein is important for its augmentation role in hepatitis B virus replication. J Virol 79: 5548-5556, 2005.

9. Yang PL, Althage A, Chung J and Chisari FV: Hydrodynamic injection of viral DNA: a mouse model of acute hepatitis B virus infection. Proc Natl Acad Sci USA 99: 13825-13830, 2002.

10. Keasler VV, Hodgson AJ, Madden CR and Slagle BL: Hepatitis B virus $\mathrm{HBx}$ protein localized to the nucleus restores $\mathrm{HBx}$-deficient virus replication in HepG2 cells and in vivo in hydrodynamicallyinjected mice. Virology 390: 122-129, 2009.

11. Newbold JE, Xin H, Tencza M, Sherman G, Dean J, Bowden S and Locarnini S: The covalently closed duplex form of the hepadnavirus genome exists in situ as a heterogeneous population of viral minichromosomes. J Virol 69: 3350-3357, 1995.

12. Gao W and Hu J: Formation of hepatitis B virus covalently closed circular DNA: removal of genome-linked protein. J Virol 81: 6164-6174, 2007.

13. Levrero M, Pollicino T, Petersen J, Belloni L, Raimondo G and Dandri M: Control of cccDNA function in hepatitis B virus infection. J Hepatol 51: 581-592, 2009.

14. Bock CT, Schwinn S, Locarnini S, Fyfe J, Manns MP, Trautwei C and Zentgraf H: Structural organization of the hepatitis B virus minichromosome. J Mol Biol 307: 183-196, 2001.

15. Pollicino T, Belloni L, Raffa G, Pediconi N, Squadrito G, Raimondo $\mathrm{G}$ and Levrero $\mathrm{M}$ : Hepatitis $\mathrm{B}$ virus replication is regulated by the acetylation status of hepatitis B virus cccDNAbound H3 and H4 histones. Gastroenterology 130: 823-837, 2006

16. Bouchard MJ, Wang L and Schneider RJ: Activation of focal adhesion kinase by hepatitis B virus HBx protein: multiple functions in viral replication. J Virol 80: 4406-4414, 2006.

17. Cougot $\mathrm{D}, \mathrm{Wu} \mathrm{Y}$, Cairo $\mathrm{S}$, et al: The hepatitis $\mathrm{B}$ virus $\mathrm{X}$ protein functionally interacts with CREB-binding protein/p300 in the regulation of CREB-mediated transcription. J Biol Chem 282: 4277-4287, 2007.

18. Harju S, McQueen KJ and Peterson KR: Chromatin structure and control of beta-like globin gene switching. Exp Biol Med (Maywood) 227: 683-700,2002.
19. Gong Q, Chen S, Guo J, et al: Chromosome remodeling related to hepatitis B virus replication in HepG2 cells. DNA Cell Biol 30: 347-354, 2011.

20. Slagle BL, Lee TH, Medina D, Finegold MJ and Butel JS: Increased sensitivity to the hepatocarcinogen diethylnitrosamine in transgenic mice carrying the hepatitis B virus X gene. Mol Carcinog 15: 261-269, 1996.

21. Belloni L, Pollicino T, De Nicola F, et al: Nuclear HBx binds the HBV minichromosome and modifies the epigenetic regulation of cccDNA function. Proc Natl Acad Sci USA 106: 19975-19979, 2009.

22. Sambrook J and Russel DW: Molecular Cloning: A Laboratory Manual. 3rd edition. Cold Spring Harbor Laboratory Press, New York, pp138-140, pp151-153, 2001.

23. Brechot C, Kremsdorf D, Soussan P, Pineau P, Dejean A, Paterlini-Brechot $\mathrm{P}$ and Tiollais $\mathrm{P}$ : Hepatitis $\mathrm{B}$ virus (HBV)related hepatocellular carcinoma (HCC): molecular mechanisms and novel paradigms. Pathol Biol (Paris) 58: 278-287, 2010.

24. Lucifora J, Arzberger S, Durantel D, et al: Hepatitis B virus X protein is essential to initiate and maintain virus replication after infection. J Hepatol 55: 996-1003, 2011.

25. Pollicino T, Squadrito G, Cerenzia G, et al: Hepatitis B virus maintains its pro-oncogenic properties in the case of occult HBV infection. Gastroenterology 126: 102-110, 2004.

26. Stoll-Becker S, Repp R, Glebe D, et al: Transcription of hepatitis B virus in peripheral blood mononuclear cells from persistently infected patients. J Virol 71: 5399-5407, 1997.

27. Hake SB, Xiao A and Allis CD: Linking the epigenetic 'language' of covalent histone modifications to cancer. Br J Cancer 90: 761-769, 2004.

28. Strahl BD and Allis CD: The language of covalent histone modifications. Nature 403: 41-45, 2000.

29. Dillon SC, Zhang X, Trievel RC and Cheng X: The SET-domain protein superfamily: protein lysine methyltransferases. Genome Biol 6: 227, 2005.

30. Hamamoto R, Furukawa Y, Morita M, et al: SMYD3 encodes a histone methyltransferase involved in the proliferation of cancer cells. Nat Cell Biol 6: 731-740, 2004.

31. Yang L, He J, Chen L and Wang G: Hepatitis B virus X protein upregulates expression of SMYD3 and C-MYC in HepG2 cells. Med Oncol 26: 445-451, 2009.

32. Park IY, Sohn BH, Yu E, et al: Aberrant epigenetic modifications in hepatocarcinogenesis induced by hepatitis $\mathrm{B}$ virus $\mathrm{X}$ protein. Gastroenterology 132: 1476-1494, 2007.

33. Rouleau J, MacLeod AR and Szyf M: Regulation of the DNA methyltransferase by the Ras-AP-1 signaling pathway. J Biol Chem 270: 1595-1601, 1995.

34. Peterson EJ, Bogler O and Taylor SM: p53-mediated repression of DNA methyltransferase 1 expression by specific DNA binding. Cancer Res 63: 6579-6582, 2003.

35. Zheng DL, Zhang L, Cheng N, et al: Epigenetic modification induced by hepatitis $\mathrm{B}$ virus $\mathrm{X}$ protein via interaction with de novo DNA methyltransferase DNMT3A. J Hepatol 50: 377-387, 2009.

36. Melegari M, Wolf SK and Schneider RJ: Hepatitis B virus DNA replication is coordinated by core protein serine phosphorylation and HBx expression. J Virol 79: 9810-9820, 2005.

37. Chadee DN, Hendzel MJ, Tylipski CP, Allis CD, Bazett-Jones DP, Wright JA and Davie JR: Increased Ser-10 phosphorylation of histone $\mathrm{H} 3$ in mitogen-stimulated and oncogene-transformed mouse fibroblasts. J Biol Chem 274: 24914-24920, 1999.

38. Min L, He B and Hui L: Mitogen-activated protein kinases in hepatocellular carcinoma development. Semin Cancer Biol 21: 10-20, 2011.

39. Wang GG, Allis CD and Chi P: Chromatin remodeling and cancer, Part I: Covalent histone modifications. Trends Mol Med 13: 363-372, 2007.

40. Hirota T, Lipp JJ, Toh BH and Peters JM: Histone H3 serine 10 phosphorylation by Aurora B causes HP1 dissociation from heterochromatin. Nature 438: 1176-1180, 2005.

41. Nowak SJ and Corces VG: Phosphorylation of histone H3: a balancing act between chromosome condensation and transcriptional activation. Trends Genet 20: 214-220, 2004.

42. Herceg Z and Paliwal A: Epigenetic mechanisms in hepatocellular carcinoma: how environmental factors influence the epigenome. Mutat Res 727: 55-61, 2011.

43. Shon JK, Shon BH, Park IY, et al: Hepatitis B virus-X protein recruits histone deacetylase 1 to repress insulin-like growth factor binding protein 3 transcription. Virus Res 139: 14-21, 2009. 